\title{
Reproducibilidad científica: ¿Qué es y por qué debemos interesarnos en geo-ciencias?
}

\author{
Daniela Ballari*
}

Recebido 8 de octubre de 2018; aceito 22 de octubre de 2018

\section{Resumen}

A pesar de que en los últimos tiempos se ha resaltado la importancia de la reproducibilidad como pilar del proceso científico, en las geo-ciencias ésta no ha sido reconocida como una preocupación inminente. Esto no es por ausencia del problema, sino por falta de evidencias que así lo demuestren. Es por ello que la comunidad científica internacional, entre ellas la relacionada con las geo-ciencias, está promoviendo el posicionamiento de la problemática y generando evidencias y propuestas que permitan superar la llamada "crisis de reproducibilidad". Así, el objetivo de este documento es aportar al avance de la discusión de la reproducibilidad científica en la comunidad científica y académica Latinoamericana de las geo-ciencias, como forma de abrir el debate crítico y propositivo sobre estrategias para mejorar la reproducibilidad de nuestras investigaciones.

Palabras clave: reproducibilidad, replicabilidad, geo-ciencias, apertura de la investigación.

\section{Resumo}

Embora a reprodutibilidade tenha sido considerada como um pilar do processo científico, nas geociências ela ainda não foi reconhecida como uma preocupação. Isto não ocorre devido à ausência do problema, mas sim devido à ausência de evidências que o demonstre. É por isso que a comunidade científica internacional, incluindo a das geociências, está promovendo a geração de evidências e diretrizes para superar a denominada "crise de reprodutibilidade". Assim, o objetivo deste artigo é contribuir para o avanço da discussão da reprodutibilidade científica na comunidade científica e acadêmica latino-americana ligada às geociências, como

* Universidad del Azuay, Av. 24 de Mayo 7-77 y Hernán Malo, Cuenca, Ecuador, correo electrónico: dballari@uazuay.edu.ec. ORCID: <https://orcid.org/0000-002-6926-4827. 
forma de abrir um debate crítico e proativo sobre estratégias para melhorar a reprodutibilidade de nossa pesquisa.

Palavras chave: reprodutibilidade, replicabilidade, geociências, abertura da pesquisa.

\begin{abstract}
Although reproducibility has been regarded as a pillar of the scientific process, in geoscience it has not been recognized as a concern yet. This is not because of the absence of the problem, but instead because of the absence of evidences. The international scientific community, including the geoscience, is promoting the generation of evidences and guidelines to overcome the "reproducibility crisis". Thus, the aim of this document is to bring to the Latin American Geoscientists the issue of scientific reproducibility, as well as to open the discussion about how we can improve the reproducibility of our research.
\end{abstract}

Key words: reproducibility, replicability, geoscience, open research.

\title{
Introducción
}

En la actualidad, al desarrollar o finalizar un proyecto de investigación, los hallazgos más relevantes son publicados en forma de artículos en revistas científicas, congresos o libros. Entre las razones para hacerlo se encuentran la necesidad de dejar un registro de la investigación para que otros puedan continuar avanzando en la misma dirección; recibir el debido reconocimiento por las ideas y los resultados; atraer el interés de otros investigadores hacia un área de conocimiento; recibir comentarios de expertos sobre las ideas y resultados documentados; y legitimar la investigación a través de la verificación independiente de métodos y resultados por parte de los revisores del artículo (Cargill y O'Connor, 2013). En ese sentido, hemos escuchado incansablemente que la descripción de los métodos en un artículo científico permite reproducir el trabajo y evaluar la validez de los resultados. Sin embargo, es evidente que en la mayoría de artículos publicados se omiten detalles metodológicos, parametrizaciones y decisiones de diseño que permitan garantizar la reproducibilidad. Por ello, los revisores y lectores encuentran dificultades para comprender los resultados, evaluar las limitaciones y, sobre todo, para reproducir el trabajo (Stark, 2018).

A pesar de ser reconocida como un pilar de la ciencia, en la práctica la reproducibilidad no es adecuadamente valorada y es difícil de implementar (Granell, Nüst, Ostermann y Sileryte, 2018). Así es que se ha generado la llamada "crisis de reproducibilidad científica", cuya importancia puede ser evidenciada de acuerdo con una encuesta a más de 1,500 científicos (Baker, 2016) en la cual el 70\% afirmó que no pudo reproducir los experimentos (publicados) de otros científicos, y el 50\% no 
pudo reproducir su propio experimento. En este último caso, no por falta de detalles metodológicos, sino por limitaciones o mal uso de la significancia estadística. Es decir, se trata del llamado p-value hacking (Head, Holman, Lanfear, Kahn y Jennions, 2015) que se caracteriza por hacer un uso inadecuado de los métodos estadísticos para encontrar y reportar selectivamente resultados y significancias favorables.

En las áreas de las ciencias de la vida y de la salud, tales como psicología, medicina, genética y biología, la preocupación por lograr la reproducibilidad científica es una preocupación reconocida e instalada desde hace algún tiempo. En las geociencias, sin embargo, no ha sido todavía reconocida como una preocupación inminente (Nüst et al., 2018). Esto no es debido a la ausencia del problema, sino por la falta de evidencias que así lo demuestren. Por ello, Nüst et al. (2018) evaluaron la reproducibilidad de un grupo de artículos obtenidos de las conferencias de la Asociación de Laboratorios de Información Geográfica de Europa (AGILE Association of Geographic Information Laboratories of Europe), una de las conferencias más destacadas en su tipo. Los resultados mostraron que, a pesar de que los autores de esos artículos reconocían la importancia de la reproducibilidad, los incentivos para hacerlo eran escasos y, por lo tanto, sus artículos no cumplían con un mínimo de reproducibilidad. A partir de este trabajo, un grupo de científicos de las geo-ciencias se encuentra impulsando acciones en pro de mejorar la transparencia y reproducibilidad de las investigaciones en áreas de las geo-ciencias y de las tecnologías de la geo-información (Gil et al., 2016; Granell et al., 2018; Konkol, Kray y Pfeiffer, 2018b, 2018a; Nüst et al., 2018; Nüst, Boettiger y Marwick, 2018; Ostermann y Granell, 2017; Shannon y Walker, 2018).

En este punto es importante regresar a la pregunta fundamental ¿Por qué quisiéramos reproducir un trabajo? Voy a direccionar la respuesta desde dos puntos de vista según el tipo de investigación.

En primer lugar están los trabajos de tipo experimental, muy comunes en las ciencias de la vida y de la salud donde existe un inminente interés de la reproducibilidad. En este caso, cuando se obtienen nuevos datos, o más extensos, los trabajos se reproducen para confirmar una vez más la validez estadística de las hipótesis. Siguiendo este planteamiento, Ostermann y Granell (2017) investigaron la reproducibilidad (usando los mismos datos y métodos) y la replicabilidad (nuevos datos y métodos similares) de información geográfica voluntaria. Sus resultados mostraron que ninguno de los trabajos investigados era reproducible y solo la mitad era replicable.

En segundo lugar están los trabajos con alto contenido computacional, comunes en las geo-ciencias. En este tipo de trabajos se manifiesta la necesidad de una reproducibilidad de tipo computacional (Konkol et al., 2018a). Es decir, es muy frecuente que se utilice software y tipos de análisis o modelizaciones muy diferentes, que requieren de la vinculación de bases de datos espaciales, sistemas de informa- 
ción geográficos, sensores remotos, modelización estadística y representación cartográfica. Así, se vuelve necesario migrar los datos de un software a otro, y de un formato a otro, para ejecutar las distintas partes del flujo de trabajo. Por consecuencia, el flujo de trabajo se vuelve complicado de reproducir. Además, cuando la interactividad con el software es manual (clicks), la reproducibilidad es incluso más complicada de realizar (Pebesma, Nüst y Bivand, 2012). Estas dificultades de reproducibilidad computacional son parcialmente superadas cuando se trabaja en un entorno de programación como puede ser R, Python o MATLAB, en los cuales se generan scripts, es decir, documentos de texto con la secuencia de funciones y órdenes que deben ejecutarse. De esta manera, todo el procedimiento se encuentra documentado en un único script que hace referencia a los datos y librerías externas a cargar, a la limpieza y pre-procesamiento de los datos, así como a las funciones específicas de análisis que deben ejecutarse en un orden determinado. A pesar que los entornos de programación facilitan el camino hacia la reproducibilidad computacional y se vinculan actualmente con los softwares más usuales en nuestra área (por ejemplo: ArcGIS, QGIS, GRASS, IDRISI, ERDAS), es necesario reconocer que su uso todavía no se encuentra lo suficientemente extendido dentro de las geociencias.

Siguiendo los lineamientos de la reproducibilidad científica se intenta motivar a los investigadores para que publiquen no solo el texto y las figuras en un artículo científico, sino también sus datos, código y flujo computacional, así como cualquier otro producto intermedio junto con sus metadatos, para así facilitar investigaciones futuras (Gil et al., 2016). A pesar que cada vez más revistas científicas permiten el envío de material suplementario como forma de facilitar la reproducibilidad, no existe ningún incentivo concreto para que los investigadores así lo hagan (Gil et al., 2016; Granell et al., 2018; Nüst et al., 2018). En este contexto, el objetivo de este documento es traer a la arena de la comunidad científica y académica Latinoamericana de las geo-ciencias la discusión sobre la reproducibilidad científica como forma de abrir el debate y cuestionamiento sobre cómo mejorar la reproducibilidad de nuestros trabajos.

El resto de este documento se organiza de la siguiente manera. Primero se provee terminología, seguido de beneficios y recomendaciones para mejorar la producibilidad de artículos en las geo-ciencias.

\section{Reproducibilidad versus Replicabilidad}

A pesar que en los últimos tiempos se ha escuchado mucho sobre la reproducibilidad científica, no es fácil encontrar una definición precisa y única. En particular existe una confusión entre Reproducibilidad y Replicabilidad. Por ello, aquí se exponen estos dos conceptos a partir de la traducción de (Barba, 2018): 
Reproducibilidad: los autores proporcionan todos los datos necesarios y los códigos para ejecutar nuevamente el trabajo, recreando así los mismos resultados.

Replicabilidad: es un estudio que llega a los mismos hallazgos científicos que otro estudio, pero recopilando nuevos datos (posiblemente con diferentes métodos) y aplicando análisis diferentes.

\section{Beneficios de la reproducibilidad}

Markowetz (2015) expresa que las razones fundamentales para invertir tiempo en reproducibilidad, (por ejemplo, "es la base de la ciencia" o "el mundo sería mejor si nuestros trabajos fueran transparentes y reproducibles"), suelen ser poco motivadores y no llevan a introducir cambios en los hábitos de trabajo y formas de conducir las investigaciones. Por ello, presentó cinco razones más pragmáticas que pueden despertar el interés en los investigadores:

1. Ayuda a evitar desastres (por ejemplo, permite detectar tempranamente errores en los análisis).

2. Hace más simple la escritura de los artículos (por ejemplo, se conocen y detallan con precisión todos los pasos metodológicos, parametrizaciones y decisiones de diseño).

3. Ayuda a los revisores a ver el artículo a la manera de los autores (por ejemplo, si se proveen los datos y el código como información suplementaria de los artículos, los revisores pueden, de manera más simple y rápida, reproducir o revisar el trabajo).

4. Permite la continuidad del trabajo (por ejemplo, nuevos ayudantes de investigación pueden continuar el trabajo que realizaron ayudantes previos, y lectores de un artículo pueden reproducir y extender el trabajo).

5. Ayuda a construir la reputación del investigador (por ejemplo, un investigador que transparenta sus datos y procedimientos generará más confianza que uno que no lo hace).

\section{Recomendaciones para mejorar la reproducibilidad en las geo-ciencias}

Desde hace tiempo se están generando recomendaciones para mejorar la reproducibilidad, con un fuerte impulso desde las ciencias de la computación. Así, Sandve et al. (2013) propusieron 10 reglas para la reproducibilidad computacional:

1. Mantener un registro de cómo se obtuvo cada resultado

2. Evitar la manipulación manual de datos

3. Documentar y archivar las versiones exactas de todos los software y librerías utilizadas

4. Controlar las versiones de todos los scripts 
5. Registrar los resultados intermedios y cuando sea posible en formatos estandarizados

6. Documentar las semillas de origen de la aleatoriedad

7. Almacenar los datos crudos u originales

8. Permitir que se inspeccionen las capas de detalle en los análisis de tipo jerárquico como, por ejemplo, los clusters jerárquico

9. Conectar declaraciones textuales con los resultados subyacentes

10. Proporcionar acceso público a las secuencias de comandos y resultados

Con un enfoque en las geo-ciencias, Gil et al. (2016) plantearon estrategias y buenas prácticas para lo que llamaron el Geoscience Paper of the Future. En él confluyen: la reproducibilidad (posibilidad de recrear los resultados de un estudio) y el linaje computacional (documentación digital sobre los datos y métodos usados para obtener los resultados). Así identificaron tres criterios básicos:

1. Los datos deben ser reutilizables a través de repositorios públicos, con documentación (metadatos), y licencias claras y citables que especifiquen las condiciones de uso.

2. El software debe ser reutilizable a través de repositorios públicos, con documentación (metadatos), y licencias claras y citables. El software incluye tanto el modelado, como la preparación y la visualización de datos.

3. El linaje computacional debe ser documentado describiendo explícitamente la serie de cálculos y sus resultados en un diagrama de flujo de trabajo de alto nivel, así como almacenado en un repositorio compartido y citable con un identificador único y persistente.

Para llevar a la práctica estos tres criterios, los autores proveen un checklist con 20 recomendaciones para guiar a los autores sobre como incluir información en los artículos de manera que facilite la reproducibilidad. Estas 20 recomendaciones se encuentran organizadas en las categorías de accesibilidad de datos, documentación de datos, accesibilidad de software, documentación de linaje, documentación de métodos, e identificación del autor. Además, se propone una hoja de ruta para que los investigadores comiencen a implementar la reproducibilidad como un hábito de trabajo, donde se expone, primero, un enfoque simple que se puede implementar en pocas horas y está dirigido a la documentación a realizar en el artículo científico. Seguido, se presenta un enfoque avanzado que, si bien requiere de una inversión considerablemente mayor de tiempo y esfuerzo, no se enfoca sólo en el artículo sino también en los hábitos de trabajo para la preparación de datos, software y linaje computacional. Por espacio no se incluyen los detalles en este documento, pero se recomienda que los lectores se dirijan al artículo mencionado (Gil et al., 2016). 
Más recientemente, Nüst et al. $(2017 ; 2018)$, propusieron la idea de "Compendio Ejecutable de Investigación - Executable Research Compendium", como una alternativa de empaquetado de datos, scripts y documentación para la reproducibilidad científica. El mismo provee una nueva forma computacional de publicación y acceso a la investigación, combinando el texto del artículo con los datos, software y documentación. La creación puede ser post-hoc, es decir, posterior a la realización de la investigación con la ayuda de un servicio y bajo la supervisión del autor; u onthe-fly donde al mismo tiempo que se realiza la investigación se produce la generación del compendio. Tras la preparación, el compendio debe ser validado, revisado y publicado. Por lo reciente de la publicación (2017 y 2018), no se han localizado publicaciones que utilicen compendios ejecutables, sin embargo, es de esperar que en el corto plazo comience a tener una aplicación efectiva.

Desde un punto de vista de la gestión de la investigación, en el marco de las conferencias AGILE <http://o2r.info/reproducible-agile/2018/>, se está impulsando la creación de guías e incentivos para que los autores comiencen a implementar prácticas de reproducibilidad. Así, se prevé proponer a AGILE nuevas guías de autores en las que se explique cómo enviar y revisar artículos reproducibles e incluso premios de conferencias para el mejor artículo reproducible. A mediano plazo, también se incentivará la reproductividad en ambientes educativos (Granell et al., 2018).

\section{Conclusiones}

La reproducibilidad no es todavía una práctica común en el ámbito científico en general, así como tampoco en las geo-ciencias. Razones para esto son la falta de evidencias que demuestren su necesidad, la falta de incentivos y la falta de guías y licencias ampliamente aceptadas y establecidas que faciliten la apertura y publicación de datos y códigos (Nüst et al., 2017). Sin embargo, se vislumbra a futuro un cambio en la configuración del artículo científico. Según la visión del "Geoscience paper of the future", ya no será un documento estático con texto y figuras, como lo es el artículo actual, sino que cada vez más será un documento dinámico e interactivo, donde los revisores y lectores podrán interactuar, re-crear y re-utilizar los datos, métodos y el código publicado junto a un artículo (Gil et al., 2016).

Esto requiere fuertes cambios a nivel individual de cada investigador. No solo debemos implementar los métodos, análisis y modelizaciones novedosas para nuestras áreas científicas, sino también formas nuevas y abiertas de documentación y publicación de las investigaciones. Por supuesto, existen razones tecnológicas y culturales que impiden la apertura y publicación de investigaciones reproducibles (Gil et al., 2016). De hecho, en ocasiones pueden existir restricciones de acceso a los datos, o co-autores con cierto rechazo a la apertura de los códigos. Por ello, las 
agencias de investigación, de financiamiento, productoras de datos, editoriales científicas y académicas tienen un rol fundamental. Deben realizar cambios en sus estructuras para promover y motivar que la reproducibilidad sea una realidad en el corto plazo, así como reconocer los esfuerzos y avances que se realicen en esta materia (Granell et al., 2018).

En el contexto Latinoamericano, la reproducibilidad científica es, sin duda, incipiente, por lo que aún no se localizan comunidades o iniciativas tendientes a divulgar y fortalecer esta práctica. Los ejemplos de reproducibilidad encontrados son aislados y parecen relacionarse con la motivación intrínseca de los propios investigadores, y no a prácticas establecidas y extendidas en la comunidad científica latinoamericana. Es por ello, que se percibe actualmente un momento adecuado para impulsar la reproducibilidad en las geo-ciencias en Latinoamérica.

Lograr que los artículos científicos sean reproducibles requiere, principalmente, de tiempo y toma de conciencia por parte de los investigadores y de las agencias vinculadas con la investigación. Por ello, es necesario introducir el tema de reproducibilidad en conferencias, en las guías de autores de las revistas científicas, en los sistemas de evaluación de la investigación e investigadores, y en cualquier otro espacio académico. La reproducibilidad debe posicionarse como un tema de preocupación y atención para la investigación latinoamericana, para así elevar los estándares científicos y caminar hacia la idea que la buena ciencia es "mostrar" y no “confiar" (Stark, 2018).

\section{Reconocimiento}

Este documento fue generado en el marco del proyecto "Representación espacial de las teleconexiones climáticas en la precipitación del Ecuador", financiado por CEDIA-Corporación Ecuatoriana para el Desarrollo de la Investigación y la Academia. Se agradece al revisor anónimo que con sus comentarios ayudó a mejorar la legibilidad y contextualización del documento.

\section{Bibliografía}

Baker, M. (2016). "1,500 scientists lift the lid on reproducibility", Nature News, 533(7604), 452.

Barba, L.A. (2018). "Terminologies for reproducible research", ArXiv Preprint ArXiv:1802.03311.

Cargill, M. and O'Connor, P. (2013). Writing scientific research articles: Strategy and steps. John Wiley \& Sons.

Gil, Y.; David, C.H.; Demir, I.; Essawy, B.T.; Fulweiler, R.W.; Goodall, J.L.; ... others (2016). "Toward the Geoscience Paper of the Future: Best practices for 
documenting and sharing research from data to software to provenance", Earth and Space Science, 3(10), 388-415.

Granell, C.; Nüst, D.; Ostermann, F.O. and Sileryte, R. (2018). Reproducible Research is like riding a bike.

Head, M.L.; Holman, L.; Lanfear, R.; Kahn, A.T. and Jennions, M.D. (2015). "The extent and consequences of p-hacking in science", PLoS Biology, 13(3), e1002106.

Konkol, M.; Kray, C. and Pfeiffer, M. (2018a). "Computational reproducibility in geoscientific papers: Insights from a series of studies with geoscientists and a reproduction study", International Journal of Geographical Information Science, 1-22.

Konkol, M.; Kray, C. and Pfeiffer, M. (2018b). The state of reproducibility in the computational geosciences.

Markowetz, F. (2015). "Five selfish reasons to work reproducibly", Genome Biology, 16(1), 274.

Nüst, D.; Boettiger, C. and Marwick, B. (2018). "How to Read a Research Compendium", ArXiv Preprint ArXiv:1806.09525.

Nüst, D.; Granell, C.; Hofer, B.; Konkol, M.; Ostermann, F.O.; Sileryte, R., and Cerutti, V. (2018). "Reproducible research and GIScience: an evaluation using AGILE conference papers", PeerJ Preprints, 6, e26561v1.

Nüst, D.; Konkol, M.; Pebesma, E.; Kray, C.; Schutzeichel, M.; Przibytzin, H. and Lorenz, J. (2017). "Opening the publication process with executable research compendia", D-Lib Magazine, 23(1/2). Retrieved from $<$ http://www.dlib.org/dlib/january17/nuest/01nuest.html>.

Ostermann, F.O. and Granell, C. (2017). "Advancing Science with VGI: Reproducibility and Replicability of Recent Studies using VGI", Transactions in GIS, 21(2), 224-237, <https://doi.org/10.1111/tgis.12195>.

Pebesma, E.; Nüst, D. and Bivand, R. (2012). "The R software environment in reproducible geoscientific research", Eos, Transactions American Geophysical Union, 93(16), 163.

Sandve, G.K.; Nekrutenko, A.; Taylor, J. and Hovig, E. (2013). "Ten simple rules for reproducible computational research", PLoS Computational Biology, 9(10), e1003285.

Shannon, J. and Walker, K. (2018). "Opening GIScience: A process-based approach", International Journal of Geographical Information Science, 1-16.

Stark, P.B. (2018). "Before reproducibility must come preproducibility", Nature, 557(7707), 613. 